\title{
55-jähriger Mann mit progredientem Hörverlust und Gangstörung
}

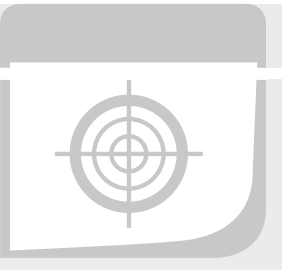

\section{Autoren}

Manuel Dafotakis ${ }^{1}$, Michael Mull ${ }^{2}$

Institute

1 Neurologische Klinik, Universitätsklinik der RWTH Aachen

2 Neuroradiologie, Universitätsklinik der RWTH Aachen
Korrespondenzadresse

Priv.-Doz. Dr. M. Dafotakis

Neurologische Klinik

Universitätsklinik der RWTH Aachen

Pauwelsstraße 30

52074 Aachen

mdafotakis@ukaachen.de

\section{Bibliografie}

DOI https://doi.org/10.1055/a-0819-5916

Klin Neurophysiol 2019; 50: 43-44

(c) Georg Thieme Verlag KG Stuttgart · New York

ISSN 1434-0275

Ein 55 jähriger Bahnbeamter stellte sich in der Ambulanz aufgrund einer sich seit 3 Jahren schleichend entwickelnden Gangstörung vor. Im Vordergrund stünde eine fehlende Koordination beim Gehen; seiner Arbeit als Zugführer könne er kaum noch nachgehen und er sei seit einem halben Jahr krankgeschrieben „wegen Rücken“. Daneben habe er eine Hörminderung bemerkt und leide immer wieder unter nackenbetonten Kopfschmerzen. Körperlich habe er abgebaut. Zudem sei eine Depression festgestellt worden, weshalb er Antidepressiva einnehme. Er rauche viel. Alkohol konsumiere er jedoch seit einem Jahr gar nicht mehr, früher habe er jedoch „schon das ein oder andere Bier gekippt“.

klinisch-neurologisch fand sich an pathologischen Befunden eine Schwerhörigkeit bds. sowie eine ausgeprägte afferent-ataktische Gangstörung mit leichter Spastik in den Beinen und pathologischem Schwanken bei Augenschluss im Romberg-Stehversuch und positive Babinski-Zeichen bds.. Die Oberflächen- und Tiefensensibilität war an den Beinen eingeschränkt. Der Knie-HackeVersuch war kaum durchführbar.

Aufgrund der Klinik wurde folgende Bildgebung angefertigt ( Abb. 1, 2), die einen pathognomonischen Befund zeigt.

\section{DIE AUFNAHMEN ZEIGEN TYPISCHE BEFUNDE}

? Welcher ist das und wie lautet Ihre Verdachtsdiagnose?

? Welche weitere Bildgebung/Untersuchungen sind notwendig?

? Wie sieht die weitere Therapie aus?

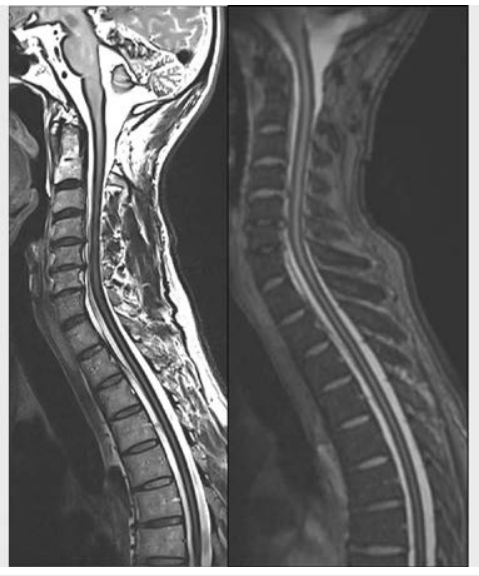

- Abb. 1 sagittale T2-gewichtete MRT-Aufnahmen zerviko-thorakal (3 T; links: T2-TSE, rechts: T2-Medic).

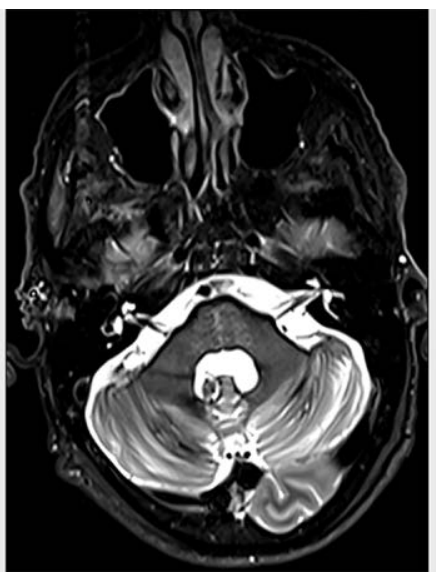

- Abb. 2 T2-gewichtetes axiales kraniales MRT (3T; T2-STIR). 


\section{Auflösung ...}

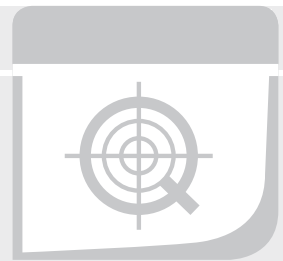

\section{Befund}

Die Aufnahmen zeigen den ausgeprägten Befund einer superfiziellen Hämosiderose des Hirnstamms und des Myelons (\Abb. 3, 4).

\section{Weitere Untersuchungen}

Bei der superfiziellen Hämosiderose müssen Blutungsquellen, die zu Hämosiderinablagerungen führen können, ausgeschlossen werden [1]. Hierzu zählen vor allem AV-Malformationen, Tumore, aber auch ein persistierendes spinales Liquorleck, welches über einen chronischen Unterdruck-Mechanismus zu rezidivierenden venösen Blutungen in den Subarachnoidalraum führen kann [2].

Zur Sicherung der Diagnose muss eine Lumbalpunktion durchgeführt werden, die typischerweise einen xantochromen Liquor zeigt. In unserem Fall fand sich ein xantochromer Liquor mit 3000 Erythrozyten und 23 Zellen $/ \mu \mathrm{l}$, das Eiweiß war auf 1,6 g/l erhöht, die Glukose normwertig und das Laktat leicht erhöht auf 3,4 mmol/l. Darüber hinaus fand sich eine schwere Schrankenstörung im ReiberDiagramm.

Bei unserem Patienten erbrachte eine CT-Myelografie den V.a. ein Liquorleck in Höhe des ventralen cervico-thorakalen Übergangs.

\section{Therapie}

Eine kausale Therapie ist dann möglich, wenn eine Ursache für die rezidivierenden subarachnoidal gelegenen Blutungen gefunden werden kann, wie z. B. durch ein spinales Liquorleck mit chronischem Unterdruck-Syndrom, sodass die Beseitigung des Lecks durch einen Blutpatch oder mittels einer neurochirurgischen Duraplastik angezeigt ist. Findet sich keine klar definierte Ursache oder aber kann diese nicht kurativ behandelt werden, so gibt es die Möglichkeit einen Heilversuch mit einem oralen Eisen-Chelatbildner (Deferiprone) zu versuchen. Erste Arbeiten weisen daraufhin, dass es sich hier um einen erfolgsversprechenden Ansatz handeln könnte [3].

\section{Interessenkonflikt}

Die Autoren geben an, dass kein Interessenkonflikt besteht.

\section{Literatur}

[1] Posti JP, Juvela S, Parkkola R et al. Three cases of superficial siderosis of the central nervous system and review of the literature. Acta Neurochir (Wien) 2011; 153: 2067-2073

[2] Kumar N. Neuroimaging in superficial siderosis: an in-depth look. AJNR Am J Neuroradiol 2010; 31: 5-14

[3] Kessler RA, Li X, Schwartz K et al. Two-year observational study of deferiprone in superficial siderosis. CNS Neurosci Ther 2018; 24: 187-192

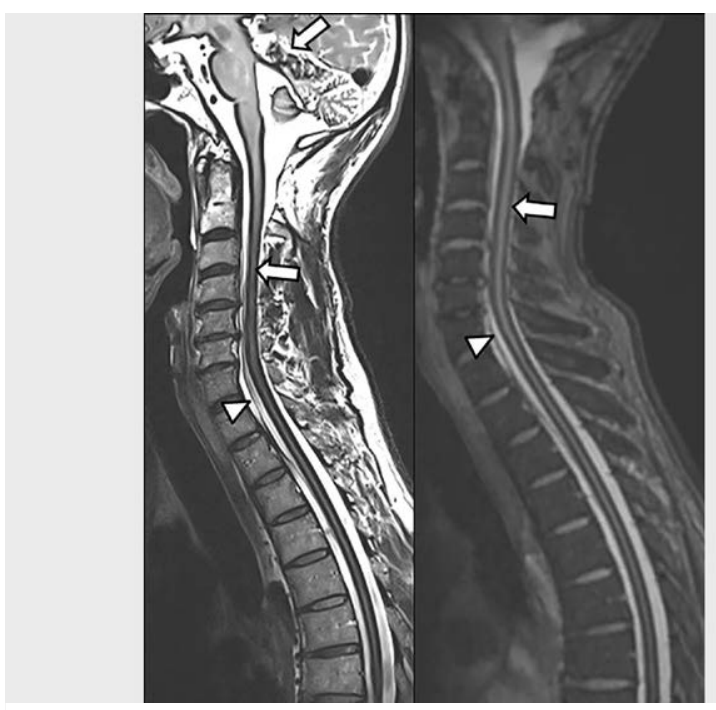

Abb. 3 Die sagittalenT2-gewichteten MRT-Aufnahmen zervikothorakal (3 T; links: T2-TSE, rechts: T2-Medic) zeigen langstreckige durch Hämosiderinablagerungen bedingte oberflächliche Signalminderungen entlang des Hals- und Brustmarks sowie eine Rückenmarksatrophie (Pfeile). Ferner ist eine extradurale Flüssigkeitskollektion ventral zervikothorakal sichtbar, hinweisend auf ein Liquorleck (Pfeilspitze).

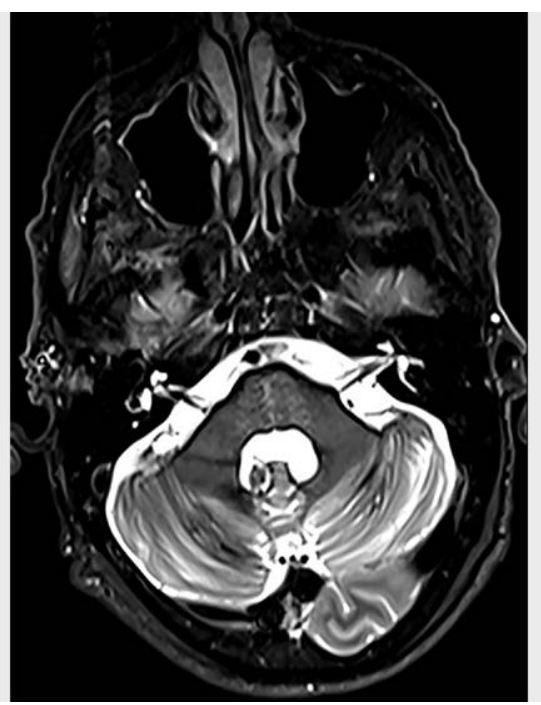

Abb. 4 Die T2-gewichteten axialen kranialen MRT-Aufnahmen (3T; T2-STIR) zeigen oberflächliche Signalminderungen zerebellär und pontin durch Hämosiderinablagerungen (Pfeile), typisch für eine Siderose. 